\title{
The SuperB Project
}

\author{
Marcello A. Giorgi*it \\ INFN and Università di Pisa, Dipartimento di Fisica "Enrico Fermi" \\ Largo B. Pontecorvo, 3 \\ E-mail: giorgi@pi.infn.it
}

The Physics motivation of the Super $B$ project is originated by the success of the the two asymmetric $e^{+} e^{-} B$ Factories, PEP-II [1] and KEKB [2],operating at the center of mass energy corresponding to the mass of the $\Upsilon(4 S)$. The initial discoveries by BABAR [3] and BELLE [4] collaboration of the indirect (2001) and the direct (2004) $C P$ violation $[5,6,7,8]$ in the $b$ sector was followed by the precise measurements of $\sin 2 \beta$, of the angles and the sides of the Unitary Triangle, with the demonstration that the $B$ sector results are fully compatible with the CKM paradigma $[9,10]$. In this presentation is shown the updated version of the parameters of $\operatorname{Super} B$ asymmetric $e^{+} e^{-}$ colliding machine, whose luminosity has a baseline value of $10^{36} \mathrm{~cm}^{-2} \mathrm{~s}^{-1}$ to integrate more than $75 a b^{-1}$ in 5 years. The design of SuperB machine [14]is strengthened by the results of the test of LNF for $D A \Phi N E$ upgrade. Some selected items of the physics program are also presented, with the attention focused on advantages given by one beam polarization higher than $80 \%$ to $\tau$ physics and by the option of run near charm threshold to the search of CP violation in charm sector. An extensive description of the general Super $B$ physics program,based in large part on b-physics is contained in CDR [11] and in various physics documents [12] [13]. Part of this bphysics program is also accessible with a lower design luminosity, at SuperKEKB and in hadron experiments as LHCb. Super $B$ is somehow the real specie evolution of PEP-II [1] and $K E K B$ [2]. Recently in 2010, before the delivery expected in 2011 of the $\operatorname{Super} B$ TDR the last comprehensive document before the construction, three intermediate reports update physics [13], accelerator [14] and detector [15] of $\operatorname{Super} B$.

35th International Conference of High Energy Physics - ICHEP2010,

July 22-28, 2010

Paris France

\footnotetext{
*Speaker.

${ }^{\dagger}$ on behalf of SuperB collaboration
} 


\section{Contents}

1. SuperB parameters 2

2. Polarization effects and charm physics 3

$2.1 \tau$ with polarization 3

2.2 Charm 4

$\begin{array}{lll}\text { 3. Conclusions } & 5\end{array}$

\section{SuperB parameters}

The table 1 contains the Super $B$ parameters with some options showing the flexibility of the machine. The same peak luminosity can be achieved with different set of parameters, the preferred option is obviously the low emittance, that allows the the luminosity of $10^{36} \mathrm{~cm}^{-2} \mathrm{~s}^{-1}$ with a minimum power consumption, allowing an obvious upgrade with maximum luminosity of 4 $10^{36} \mathrm{~cm}^{-2} \mathrm{~s}^{-1}$. The third column contains the parameters for running near charm threshold.

Table 1: Machine parameters for Super $B$

\begin{tabular}{lcccc}
\hline \hline Parameter & units & Baseline & Low Emittance & $\tau$-charm \\
\hline Energy (HER/LER) & $\mathrm{GeV}$ & $6.7 / 4.18$ & $6.7 / 4.18$ & $2.58 / 1.61$ \\
Circumference & $\mathrm{m}$ & 1258 & 1258 & 1258 \\
Luminosity & $10^{36} \mathrm{~cm}^{-} 2 \mathrm{~s}^{-1}$ & 1.0 & 1.0 & 0.1 \\
Beam Current (HER/LER) & $\mathrm{A}$ & $1.89 / 2.41$ & $1.46 / 1.80$ & $1.36 / 1.70$ \\
$\mathrm{~N}_{\text {bunches }}$ & & 978 & 978 & 1956 \\
$\varepsilon_{y}(\mathrm{HER} / \mathrm{LER})$ & $\mathrm{pm}$ & $5 / 5.8$ & $2.5 / 2.9$ & $13 / 15.75$ \\
$\varepsilon_{x}($ withIBS) $(\mathrm{HER} / \mathrm{LER})$ & $\mathrm{nm}$ & $2 / 2.41$ & $1 / 1.21$ & $5.2 / 6.3$ \\
$\beta_{y}$ (HER/LER) & $\mu \mathrm{m}$ & $252 / 206$ & $179 / 146$ & $658 / 536$ \\
$\beta_{x}$ (HER/LER) & $\mathrm{cm}$ & $2.6 / 3.2$ & $2.6 / 3.2$ & $6.76 / 8.32$ \\
Crossing angle $\chi$ & $\mathrm{mrad}$ & 60 & 60 & 60 \\
One beam Polarization & $\%$ & 85 & 85 & \\
RF power (AC line) & $\mathrm{MW}$ & 17.1 & 12.7 & 1.68 \\
hor. tune shift (HER/LER) & $\%$ & $0.0021 / 0.0033$ & $0.0016 / 0.0025$ & $0.0052 / 0.0060$ \\
ver. tune shift (HER/LER) & $\%$ & $0.0978 / 0.0901$ & $0.0901 / 0.0901$ & $0.0914 / 0.0915$
\end{tabular}




\section{Polarization effects and charm physics}

\section{$2.1 \tau$ with polarization}

The option of one beam $80 \%$ polarized increases its discovery potential of Super $B$, allowing a new way of measuring the Standard Model (SM) and giving access to the CP violation study in $\tau$ sector,to the investigation of $\tau$ magnetic structure in addition to parameters and facilitating the study Lepton Flavor Violation (LFV). The present limits to branching fraction for some LFV decays have been established by BABAR $[16,17]$ and BELLE $[18,19]$ :

$\operatorname{BABAR}(\tau \rightarrow \mu \gamma)=4.410^{-8}$ with $515 \mathrm{fb}^{-1}$ and $(\tau \rightarrow l l l)=1.8-3.3 \quad 10^{-8}$ with $468 \mathrm{fb}^{-1}$ and $\operatorname{BELLE}(\tau \rightarrow \mu \gamma)=4.510^{-8}$ with $535 \mathrm{fb}^{-1}$ and $(\tau \rightarrow l l l)=1.5-2.710^{-8}$ with $782 \mathrm{fb}^{-1}$.

An optimized analysis based on the most recent results from BABAR for $\tau \rightarrow \ell \ell \ell$ has been extrapolated to Super $B$ for $75 \mathrm{ab}^{-1}$.

In absence of signal events the extrapolated UL ( $90 \% \mathrm{CL}$ ) lays in the range $2.3 \times 10^{-10}$ to $8.2 \times 10^{-10}$, depending on the channel. The $3 \sigma$ evidence of a branching fraction are between $1.2 \times 10^{-9}$ and $4.0 \times 10^{-9}$. The extrapolation from BABAR to $\operatorname{Super} B$ based on this analysis is shown in Fig. 1.

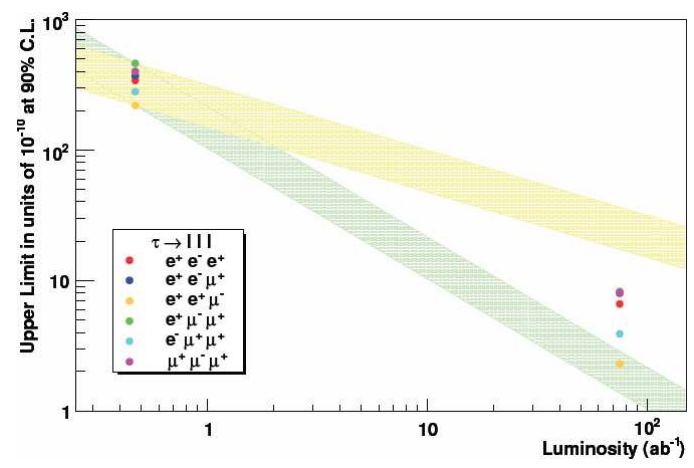

Figure 1: The limits for various LFV channels versus the integrated luminosity are not strongly affected by systematic errors, they scale better than $\sqrt{(} L)$

LFV in $\tau \rightarrow \mu \gamma$ is limited by background, whose main sources are $\tau \rightarrow \mu \nu \bar{v} \gamma$ and the wrong combination of one $\mu$ with a $\gamma$ from initial state radiation. The production of polarized $\tau$ 's allows the reduction of the "irreducible" background from $\tau \rightarrow \mu v \bar{v} \gamma$, improving the (signal)/(background)ratio by almost a factor of 2 as evident in Fig. 2. If LFV would be found the polarization is powerful tool for studying the helicity structure of the violating coupling. The polarization as discussed in [12] is also a powerful tool in $\tau$ physics for EDM search, for CP/T violation in decay and for the investigation the $\tau$ magnetic structure.

Precise tests in the SM electroweak sector are also available through beam polarization. SLD and LEP experiments measured $\sin ^{2} \theta_{W}$ and the neutral current coupling $Z-b \bar{b}$, using lepton production asymmetries. Super $B$ will be able to record $\sim 30 \times 10^{6}$ hadronic $\mathrm{Z}$ virtual decays, which should be compared to the $0.5 \times 10^{6}$ recorded by SLC. If the polarization is under control at the level $\mathrm{O}(0.5 \%)$, the error on $\sin ^{2} \theta_{W}$ will be reduced of almost a factor 2,in energy regions far below the $\mathrm{Z}$ pole.The study Z- $b \bar{b}$ coupling, with the error on $g_{V}^{b}$ reduced by a factor 8 is also possible. 

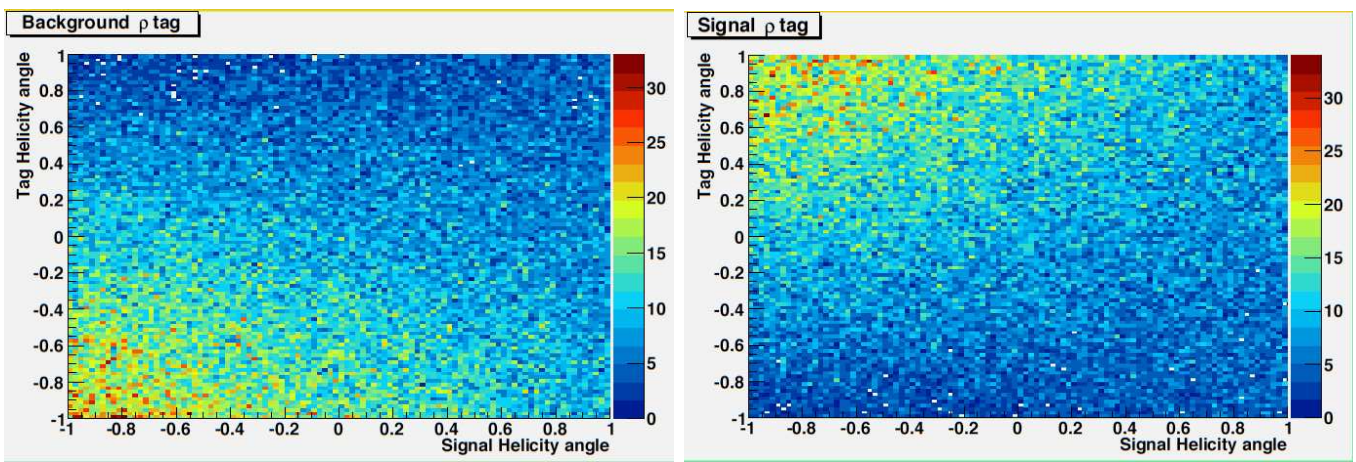

Figure 2: The helicity angle is defined as the angle between the flight direction of the track in the $\tau$ rest frame and the $\tau$ direction in the lab frame. The correlation between signal and tag helicity angles is shown for $\rho$-tagged events both in the case of backgrounds (left) and signal (right).

Then the deviation from SM observed by LEP and SLD may be eiher confirmed (hence implying $\mathrm{NP}$ ) or rejected (proving the robustness of the SM).

\subsection{Charm}

The recent observation of large $D^{0} \bar{D}^{0}$ mixing, in the experiments BABAR [21] and BELLE [22] at B-Factories but also CDF [23] raises the exciting possibility of finding $C P$ violation in charm decay, which would almost certainly indicates physics beyond the Standard Model. At present no evidence is shown of $C P$ violation as can be clearly seen from the HFAG [24] average.The HFAG fits to the measured oservables for $\mathrm{x}, \mathrm{y}$ exclude the no-mixing point $(\mathrm{x}=\mathrm{y}=0)$ at $6.7 \sigma$ and don't show any evidence for $C P$ violation. The resulting central values at $68.3 \%$ C.L. and the intervals 95 \% C.L.,are: $x=0.97_{-0.29}^{+0.27}(0.39-1.48$ at $95 \% C L), y=0.78_{-0.19}^{+0.18}(0.41-1.13$ at $95 \% C L)$ showing no evidence of $C P$ violation in $D^{0}-\bar{D}^{0}$ violation. The expected overall uncertainties on the mixing parameters with $75 a b^{-1}$ of SuperB are $4.910^{-4}$ for $\mathrm{x}$ and $3.510^{-4}$ for $\mathrm{y}$ and on the $C P$ parameters $1.910^{-4}$ for $\varepsilon$ and 1.9 degrees for $\operatorname{Arg} \frac{q}{p}$. Runs with lower background at open charm threshold in SuperB would allow the measurement of both direct and indirect CPV. Figure 3 shows the situation with $\operatorname{Super} B$ five years run in three cases.
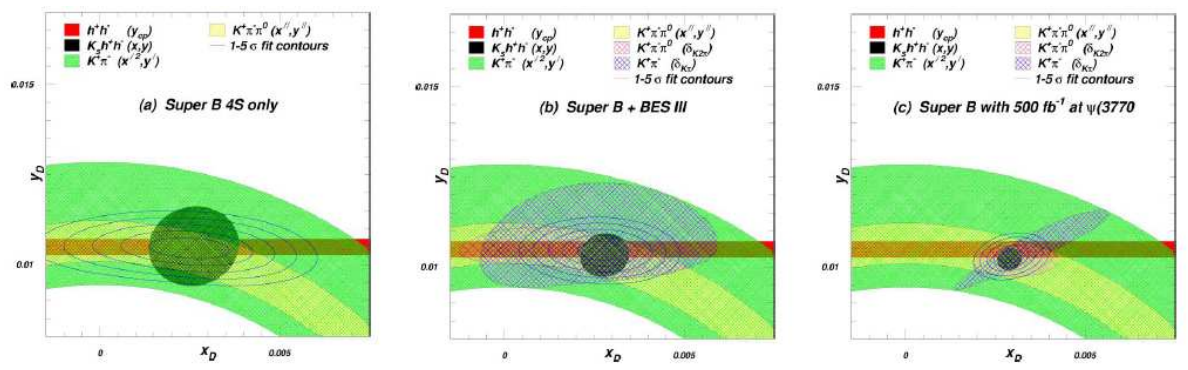

Figure 3: Discovery potential of $\operatorname{Super} B$ in charm sector. The expected (x,y) plot from $\operatorname{Super} B$ data collected at $\Upsilon(4 \mathrm{~S})(\mathrm{a})$, from $\operatorname{Super} B$ data combined with results expected BES III (b) and from $\operatorname{Super} B$ with data at $\Upsilon(4 \mathrm{~S})$ plus data $\left(500 \mathrm{fb}^{-1}\right)$ collected at charm threshold. 


\section{Conclusions}

In 5 years after the beginning of the construction is planned the start of data taking with a robust apparatus derived from the concepts of BABAR Many components of BABAR detector will be re-used in $\operatorname{Super} B$, possible upgrades of the vertex detector to extremely high resolution is under evaluation to allow the study of $C P$ violation in charm with time dependent analysis in runs near charm threshold. The SuperB project has received many encouraging signals coming from the Italian Government. Tough still not funded, the project and the machine design are now in the TDR phase and the document will be delivered in 2011, its validity has received so far important recognition. The management structure of $\operatorname{Super} B$ is in place and the program for recruiting the personnel for the machine and detector construction has started.

\section{References}

[1] “PEP-II: An Asymmetric B Factory. Conceptual Design Report. June 1993," SLAC-R-418.

[2] “KEKB B-Factory Design Report”, KEK Report 95-7, 1995.

[3] B. Aubert et al. [BABAR Collaboration], Nucl. Instrum. Meth. A 479, 1 (2002)

[4] K. Abe et al. [Belle Collaboration], Nucl. Instrum. Meth. A 479, 117 (2002).

[5] B. Aubert et al. [BABAR Collaboration], Phys. Rev. Lett. 87, 091801 (2001)

[6] K. Abe et al. [Belle Collaboration], Phys. Rev. Lett. 87, 091802 (2001)

[7] B. Aubert et al. [BaBar Collaboration], Phys. Rev. Lett. 93, 131801 (2004)

[8] K. Abe et al. [Belle Collaboration], Phys. Rev. Lett. 93, 021601 (2004)

[9] N. Cabibbo, Phys. ReV. Lett. 10, 531 (1963)

[10] M. Kobayashi and T. Maskawa, Prog. Theor. Phys. 49, 652 (1973)

[11] M. Bona et al., arXiv:0709.0451 [hep-ex].

[12] Proceeding of SuperB Workshop VI: arXiv:0810.1312v1 [hep.ex]

[13] B.O'Leary et al. arXiv:1008.1541v1

[14] M.E.Biagini et al. arXiv:1009.6178v1

[15] E.Grauges et al. arXiv:1007.4241v1

[16] B. Aubert et al. [BaBar Collaboration] Phys. Rev. Lett. 95, 041802 (2005)

[17] B. Aubert et al. [BaBar Collaboration] Phys. Rev. Lett. 98, 251803 (2007)

[18] K. Hayasaka et al. [Belle Collaboraton] arXiv:0705.0650v1[hep-ex]

[19] Y.Miyazaki et al. [Belle Collaboraton] Phys. Lett. 660, 154 (2008)

[20] B. C. Allanach et al., arXiv:hep-ph/0202233.

[21] B. Aubert et al. [BaBar Collaboration] Phys. Rev. Lett. 98, 211802 (2007). B. Aubert et al. [BaBar Collaboration],arXiv:0712.2249 [hep-ex]- submitted to Phys. Rev. D.

[22] M.Staric et al. [Belle Collaboraton] Phys. Rev. Lett. 98, 211803 (2007).

[23] T.Aaltonen et al. [CDF Collaboraton]Phys. Rev. Lett. 100, 121802 (2008).

A.J.Schwartz,Workshop on Charm Physics, Beijing 26-27 November 2007. arXiv:0803.0082 [hep-ex].

[24] HFAG (charm subgroup) http://www.slac.stanford.edu/xorg/hfag/charm/. 